\title{
Attention and auditory evoked responses to low-detectability signals ${ }^{1}$
}

TRUMAN E. MAST ${ }^{2}$ AND CHARLES S. WATSON

CENTRAL INSTITUTE FOR THE DEAF, St. Louis, Mo. 63110

Comparisons were made between cortical evoked responses obtained under two conditions: (1) while Ss were reading, and (2) while they were attempting to count auditory signals. The amplitudes of evoked responses to low-detectability atrditory stimuli were found to be approximately doubled when the Ss were required to count the number of stimuli, as compared to amplitudes recorded when they were reading. The duration of the response was also markedly increased. These increases in response amplitude and duration are considerably greater than those observed in earlier experiments, where high-level signals were used. Inter-S variability of the waveform of the average evoked response was observed to be much less when the Ss counted the stimuli. In another experiment the level of the auditory signal was varied over a range of approximately +4 to -4 decibels relative to the listeners' behavioral thresholds. The per cent of signals which they counted varied from near-zero to 100 , over this range, and the evoked response concurrently showed a variation from "unmeasureable" to approximately 8 microvolts.

The effects of attention on human evoked responses have been explored by a number of investigators. Several different techniques for "focussing" attention have been used: requiring the observers to respond to some change in the stimulus (Davis, 1964; Satterfield, 1965; Debecker \& Desmedt, 1966), using a distracting background (Donchin \& Cohen, 1967), presenting stimuli in a vigilance task (Spong et al, 1965), giving a psychological significance to the stimuli (Larson, 1960; Chapman \& Bragdon, 1964), or counting the stimuli (Davis \& Yoshie, 1963; Garcia-Aust et al, 1965; Gross et al, 1965). These experiments are in general agreement that an increase of attention to the stimulus causes an enhancement of the evoked response. However, most of these studies have concentrated on relatively high-intensity stimuli, and have frequently shown only small enhancements with increased attention. In particular, the two papers dealing with counting of high-intensity stimuli show only small effects (Davis \& Yoshie, 1963; Gross et al, 1965).

The growing literature on the evoked response to auditory stimuli, especially with regard to the development of practical audiometric procedures, generally describes these responses as being clear and measurable only when the auditory stimulus exceeds about $10 \mathrm{~dB}$ sensation level (Goldstein, 1967). The experiments reported here were designed to examine evoked responses to auditory stimuli, the levels of which were nearer to behavioral thresholds. We were also interested in learning whether the techniques for enhancing the evoked response, as described above, might be effective in producing measurable response to such faint sounds. The "counting" procedure for focussing attention was selected, as it seemed uniquely suited to the requirements of evoked response measurements. If a random number of stimuli, or "signals," is presented in a listening period, then the listener can afterward be queried for his "count," thus permitting an evaluation of the S's performance. In addition, the counting procedure does not involve an overt response, such as pressing a button after each signal heard, and thus should avoid some of the complications of the cortical electrical activity known to accompany movement (Bates, 1951).

\section{METHOD}

Ss were three men and two women, ranging in age from 16 to 25 years.

Stimuli were $1000-\mathrm{Hz}$ tone bursts each with a total duration of $110 \mathrm{msec}$ including rise and fall times of $10 \mathrm{msec}$. They were

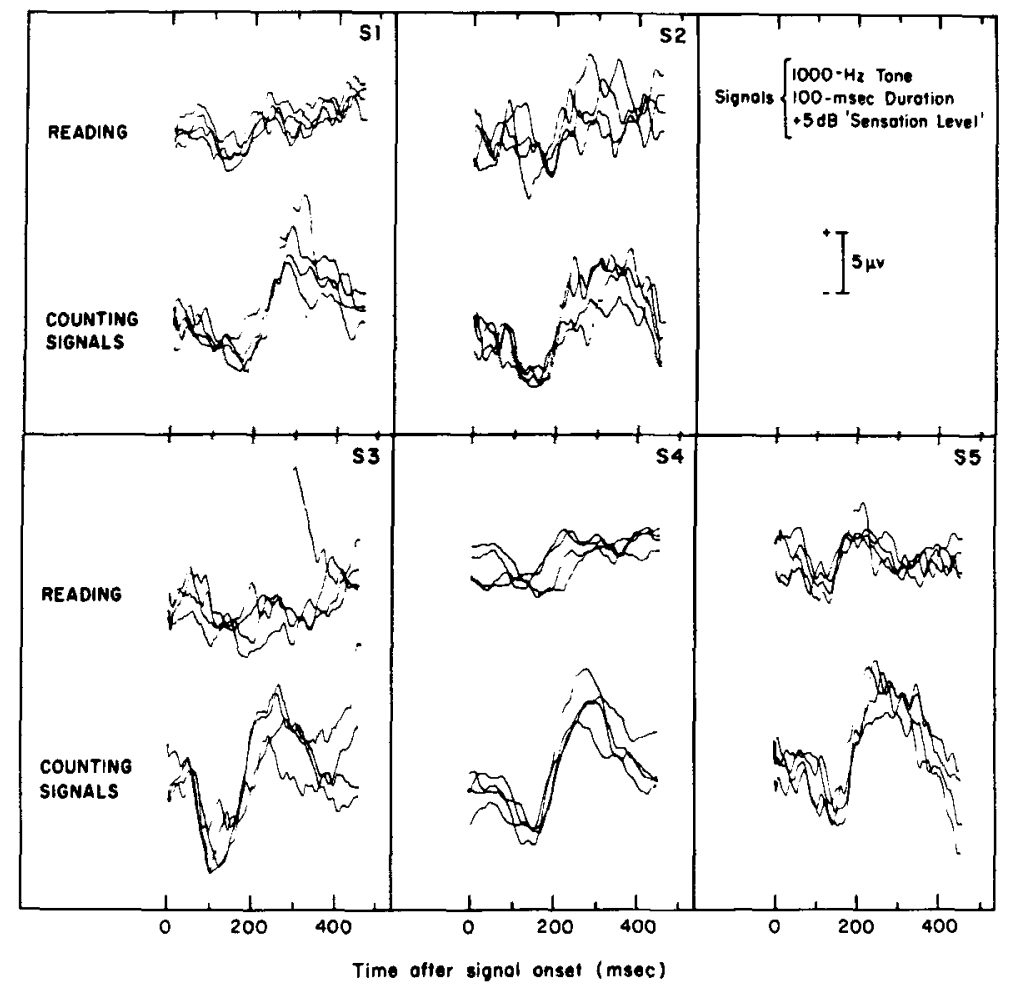

Fig. 1. Comparison of evoked responses obtained from five $S s$ during conditions of reading and counting. The number of responses averaged for each trace varies from 42 to 65 , but the numbers for the traces in the two conditions are always similar. The voltage calibration is for 50 responses. In this and subsequent figures positivity at the vertex is plotted upward. 
presented to the Ss monaurally (right ear) through TDH39 earphones mounted in Rudmose Otocups. Ss were seated in a quiet room separate from the experimental equipment. Stimuli were controlled by a LINC computer. A random sequence of signal and no-signal trials was presented at a regular rate of one every 1.5 sec. A block of such stimuli consisted of 100 trials, with a priori signal probability equal to 0.5 .

Recording electrodes were placed on the vertex with the earlobe used as a reference. Electrical activity was amplified on a Grass P511 amplifier with a pass band of 0.3 to either 500 or $2000 \mathrm{~Hz}$. Responses were averaged by the LINC computer with a sample rate of $1 / 3.5 \mathrm{msec}$. Averages were made of the response over the $450 \mathrm{msec}$ following stimulus onșet. In those trials where no stimulus was given, a separate control average was accumulated. Data were stored on digital magnetic tape and the responses (averages) from several blocks of 100 presentations were usually grouped together.

\section{RESULTS}

Preliminary experiments were done to determine whether evoked cortical potentials could be obtained in response to auditory signals at or below behavioral threshold. No consistent evoked responses were obtained when Ss were either reading or attempting to count such stimuli. With stimuli of an intensity approximately $5 \mathrm{~dB}$ above behavioral threshold (by method of adjustment) consistent responses were obtained when Ss were attempting to count the number of signals presented to them, and usually, responses were also observed when they were reading.

Five Ss were used in the comparison of responses during reading and counting. A method-of-adjustment threshold was obtained for each $\mathrm{S}$ for the $110 \mathrm{msec} 1000-\mathrm{Hz}$ tone bursts we used as stimuli. The stimuli were then presented in a signal and no-signal random sequence as described above. During the trials, Ss were given material to read, and were told that they would be quizzed on the material at the end of the session.

Four or five blocks of 100 presentations were given while the $S$ was reading. Following the measurements with the $S$ s reading, they were required to count the number. of signals in blocks of 100 presentations. The Ss reported the number of signals counted after the completion of each block, and they were then told how many signals had in fact been presented. Again, four or five blocks were given.

The results are shown in Fig. 1. In every case there is a marked increase in amplitude during counting. Mean amplitudes over all Ss during counting were 2.2 times those recorded during reading. (Amplitudes were measured from the peak of $N_{1}$ to the peak of $P_{2} . N_{1}$ is the negative peak at roughly $100 \mathrm{msec}$ and $P_{2}$ the positive peak at about 175 msec.) During counting, the responses also show more consistent waveforms, and longer durations of the late positive component.

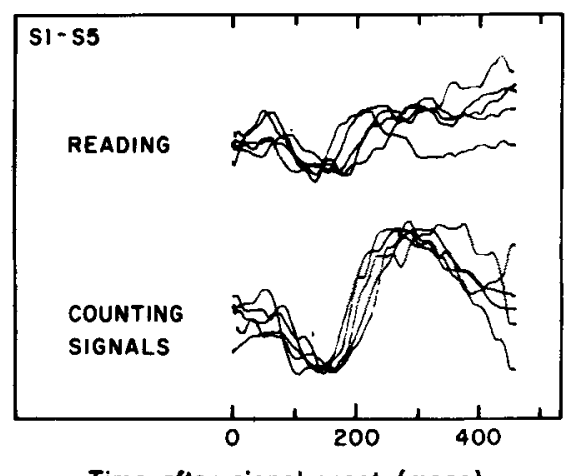

Time ofter signal onset (msec)

Fig. 2. Waveform comparisons for the evoked responses from the five Ss shown in Fig. 1. Each trace is the average of the traces for a single $S$ in Fig. 1. The gain has been adjusted so that equal $N_{1}-P_{2}$ amplitudes are represented for each $S$. The averaged ratio of counting and reading amplitudes has been maintained.

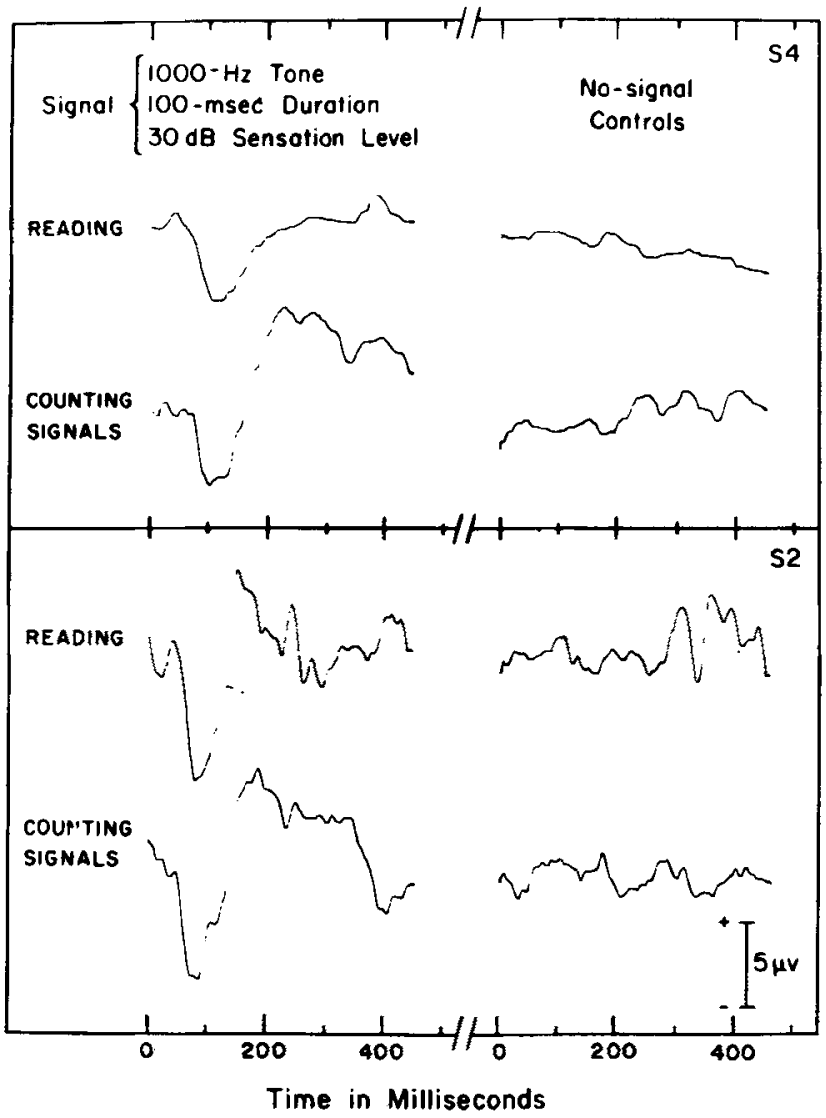

Fig. 3. Ewoked responses and control averages from two Ss under conditions of reading and counting. Note signal level is higher than that used for responses shown in Figs. 1 and 2.

Figure 2 shows that waveforms from different subjects are very similar in the counting condition although considerable variability is obtained when they are reading. In this figure, gain was adjusted to yield equal amplitudes $\left(\mathrm{N}_{1}-\mathrm{P}_{2}\right)$ for all Ss. The consistent prolongation of $P_{2}$ found when $S s$ are counting is also apparent in this figure.

In another experiment, we tested two Ss to determine if the effect of counting vs reading was also present at higher stimulus intensities. Figure 3 shows that one case showed a slightly higher $N_{1}-P_{2}$ amplitude when reading and the other had no noticeable increase. However, in both cases, there is a definite prolongation of $\mathbf{P}_{\mathbf{2}}$.

In the last experiment reported here, the procedure was modified to determine how near to behavioral "threshold" we could obtain evoked responses. In these cases, signal and no-signal presentations were not made but instead signals having two different levels were presented in random sequence. The $S$ was instructed to count all signals that he heard. Pairs of signal levels, separated by either 5.0 or $7.5 \mathrm{~dB}$, were used in each block of trials. The higher level signal was always at or above the behavioral threshold that had been obtained during the same session. The lower level signal was below that threshold.

The percentages of signals counted under this procedure are shown in Fig. 4 using a rather drastic simplifying assumption to estimate the number of signals counted at each signal level. The assumption is that when the S's "count" was larger than the number of higher level signals, this "count" could be treated as including all of the high-level signals. The total number counted, minus the number of high-level signals, then represents the estimated number of low-level signals counted. 

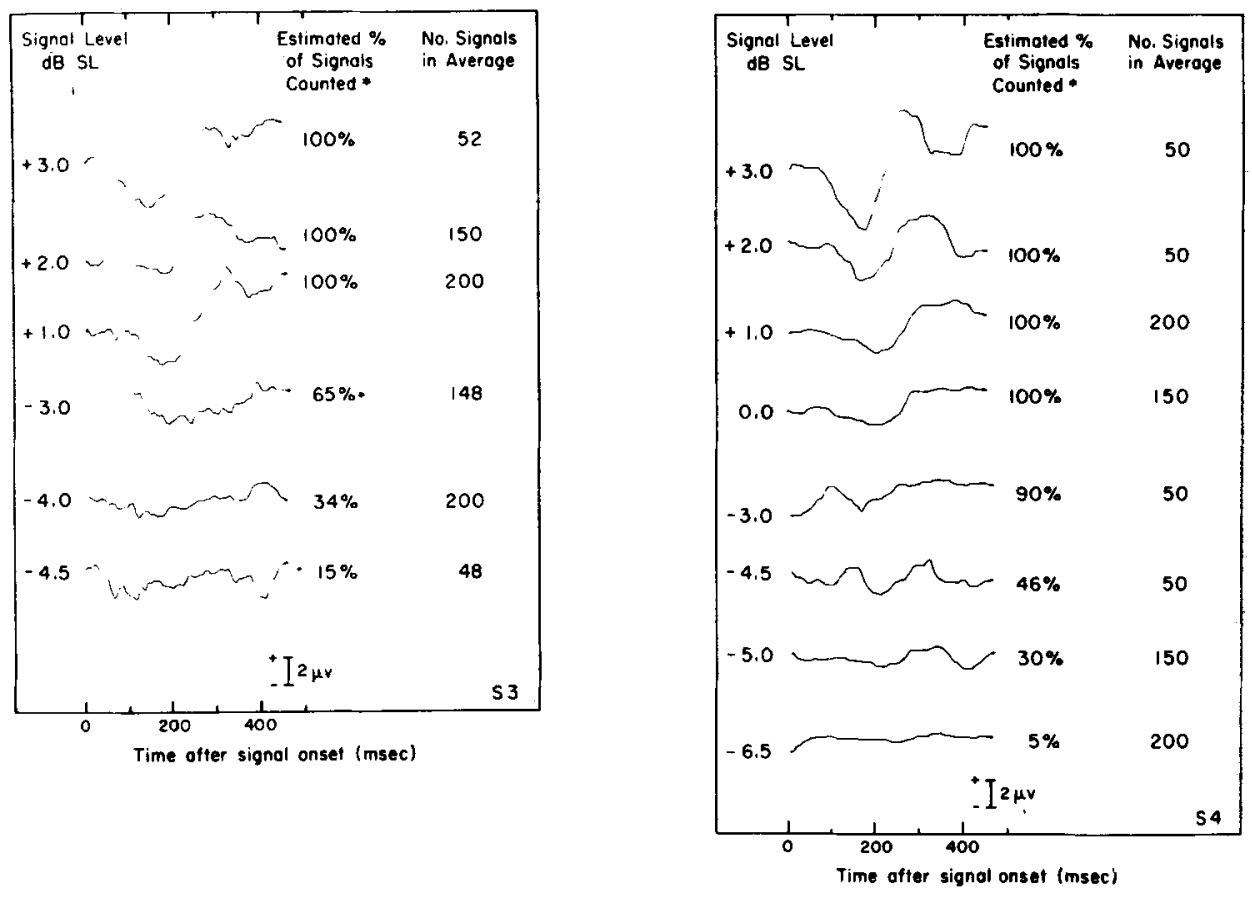

This assumption ignores the fact that neither the state of a listener nor the trial-by-trial strength of the signals are as constant as it would require. But the psychometric functions displayed in Fig. 5 are remarkably steep in comparison with typical slopes reported in detection research (Green \& Swets, 1966), suggesting that variability of either $S$ or stimulus has not been a serious problem.

Separate average evoked responses were collected for each of the signal levels used in this experiment. Figure 5 shows these records, and also the associated estimates of the number of signals counted at each level. Estimated "counts" of $100 \%$ are associated with clear evoked responses, and the electrical response disappears at about the same signal levels as does the behavioral measure.

The pairing of two levels of signal in each block complicates the analysis of this experiment, especially if $2-t_{0}-3-\mathrm{dB}$ effects are of interest. However, our experience suggests that it is difficult to conduct such an experiment with a single signal level in each blcck. When only very weak signals are used, the listeners report that they tend to forget what frequency it is that they are trying to hear and these reports are often associated with a general breakdown of performance. An adequate design can probably avoid both this problem and the need for the assumption introduced above, but we doubt that the results shown in Figs. 4 and 5 will be greatly affected by any small modification of the procedure.

\section{SUMMARY AND DISCUSSION}

We have demonstrated that consistent responses to low-level auditory stimuli (within a few decibels of behavioral threshold) can be readily obtained when the $S$ focusses attention on the stimuli, that is, when he is instructed to remain physically quiet, but to count them. Previously it has been reported that counting made insignificant differences in auditory evoked responses (Davis $\&$ Yoshie, 1963) or that counting increased amplitude by only some 10-15\% (Gross et al, 1965). These two papers described research with rather high-level signals and thus the S's task was an easy one. Davis (1964) reports that performing an auditory discrimination results in an increased evoked potential amplitude, but that the task must be a difficult one. An easy task was not sufficient to demonstrate the effect.

In the present experiment large increases in evoked response amplitude accompany the difficult task of counting auditory

signals when they are presented at levels close to behavioral threshold. The increase is of the order of a $100-150 \%$ change of the $N_{1}-P_{2}$ portion of the response, for signal levels close to behavioral threshold, compared to the response observed with a passive (reading) S. Enhancement of the amplitude of the response by counting may or may not be apparent when the signal level is raised 25 to $30 \mathrm{~dB}$ above these levels.

A late component, or possibly an extension in time of the second positive $\left(\mathrm{P}_{2}\right)$, is consistently present when the listener attempts to count, both for signals close to and $25-30 \mathrm{~dB}$ above behavioral threshold. A late positive component of the cortical response has been reported in other experiments when the $S$ has high incentive to respond after a stimulus (Wilkinson \& Morlock, 1966; Donchin \& Lindsley, 1966; Bates, 1951). But these latter experiments involved overt behavioral responses by the $S$ and the late component of the evoked response was definitely in the form of an additional wave $\left(P_{3}\right)$. Our results may thus differ from experiments in which overt behavioral responses are used.

We believe one of the most interesting results of these experiments to be the relation between the dynamic ranges of the physiological response (averaged evoked response) and of the behavioral performance (ability to count the signals). As shown in Figs. 4 and 5 , these two responses range from essentially "unmeasurable" to "strong-and-clear" over a range of no more than $7 \mathrm{~dB}$, and their ranges appear to be approximately coincident. Such close correspondence between a behavioral and a physiological measure is fairly unusual in our experience. Exactly how this relation should be interpreted is not clear, since recent developments in signal detection research (e.g., Green \& Swets, 1966; Watson, Franks, \& Hood, 1967) suggest that auditory signals are detectable in forced-choice procedures considerably below the signal levels referred to here as "behavioral threshold."

We are considering two alternative explanations. One is that the evoked response may be only indirectly related to the degree of activity in a sensory channel, but rather be a function of the amount by which the input has exceeded the listener's criterion. (Thus it might be the change of criterion which distinguishes between the "reading" and "counting" conditions.) The other possibility is that our conclusion is primarily dependent on the number of evoked responses which are included in each average. That is, we may be committing the error that many interpretations of averaged physiological potentials are subject to. The lowest 


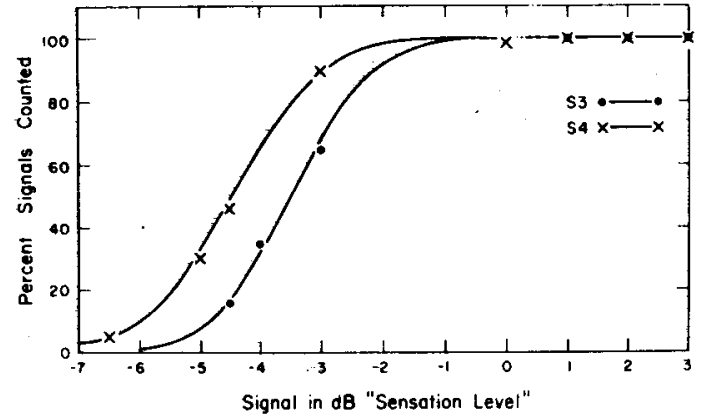

Fig. 5. "Psychometric function" obtained from data shown in Fig. 4. (See text for explanation of ordinate.)

stimulus level at which a particular averaged response can be demonstrated depends on the number of individual responses over which the average is taken. When this number increases, the signal-noise ratio in the average also increases and the averaged response may be detected at a lower stimulus level. In our case, the evoked response can be expected to diminish in amplitude at the lower stimulus intensities while the background EEG activity remains constant. Therefore our signal-noise ratio (evoked response-EEG background ratio) will be quite low for "subthreshold" stimuli. It may be this factor rather than a genuine absence of evoked response that prevents our demonstration of a response at our lowest signal levels.

In a recent study Onishi and Davis (1968) have demonstrated that the vertex potential, unlike psychophysical responses, is mainly related to the intensity of an auditory signal during its first 30 msec. One of these authors (Davis, 1968) has observed that this failure of the typical time-intensity trading relation, coupled with the data of the present experiment, may predict that the evoked response could be detected at signal levels below behavioral threshold.

The present authors, however suspect that only a very slight vertex response will be recorded below signal levels which lead to a behavioral response. This view is consistent with our earlier discussion, inasmuch as the evoked response as well as the "perception" may together be dependent on the listener's criterion. Thus, the usual procedures for shifting criterion might likewise shift the level of signal at which the vertex potential is first observed. With Davis, we believe that this issue is worth putting to experimental test.

\section{REFERENCES}

BATES, J. A. Electrical activity of the cortex accompanying movement. $J$. Physiol., (Lond.) 1951, 113, 240-257.

CHAPMAN, R. M., \& BRAGDON, H. R. Evoked responses to numerical and non-numerical visual stimuli while problem solving. Nature, (Lond.) 1964, 203, $1155-1157$.
DAVIS, $H$. Enhancement of evoked cortical potentials in humans related to a task requiring a decision. Science, 1964, 145, 182-183.

DAVIS, H., \& YOSHIE, N. Human evoked cortical responses to auditory stimuli. Physiol, 1963, 6, 164.

DAVIS, H. Personal communication, 1968.

DEBECKER, J., \& DESMEDT, J. S. Rate of intramodality switching disclosed by sensory evoked potentials averaged during signal detection tests. J. Physiol, (Lond.) 1966, 185, 52-82.

DONCHIN, E., \& COHEN, L. Averaged evoked potentials and intramodality selective attention. EEG clin. Neurophysiol., 1967, 22, 537-546.

DONCHIN, E., \& LINDSLEY, D. B. Averaged evoked potentials and reaction time to visual stimuli. EEG clin. Neurophysiol., 1966, 20, 217-223.

GARCIA-AUST, E., BOZACZ, H., \& NANZULLI, A. Effects of attention upon visual evoked responses. EEG clin. Neurophysiol. 1964, 17, 136-143.

GOLDSTEIN, R. Electroencephalic audiometry. In A. Bruce Graham (Ed.), Sensorineural hearing processes and disorders. Boston: Little, Brown, and Company, 1967.

GREEN, D. M., \& SWETS, J. A. Signal detection theory and psychophysics. New York: Wiley, 1966.

GROSS, M. M., BEGLEITER, H., TOBIN, M., \& KISSIN, B. Auditory evoked response comparison during counting clicks and reading. EEG clin. Neurophysiol, 1965, 18, 451-454.

JANE, J., SMIRNOV, G., \& JASPER, H. Effects of distraction upon simultaneous visual and auditory evoked potentials. EEG clin. Neurophysiol., 1962, 14, 344-358.

LARSON, L. Correlation between the psychological significance of stimuli and the magnitudes of the startle blink and evoked EEG potentials in man. Acta physiol. Scand., 1960, 48, 276-294.

ONISHI, S., \& DAVIS, H. Effects of duration and rise time of tone bursts on evoked V potentials. J. Acoust. Soc. Amer., 1968, in press.

SATTERFIELD, J. H. Evoked cortical response enhancement and attention in man. A study of responses to auditory and shock stimuli. $E E G$ clin. Neurophysiol, 1967, 19, 470-475.

SPONG, P., HAIDER, M., \& LINDSLEY, D. B. Selective attentiveness and cortical evoked responses to visual and auditory stimuli. Science, 1965, 148, 395-397.

WATSON, C. S., FRANKS, J. R., \& HOOD, D. C. Detection of tones in the absence of external masking noise. J. Acoust. Soc. Amer., 1967, 42, 1194.

WILKINSON, R., \& MORLOCK, H. Auditory evoked response and reaction time. EEG clin. Neurophysiol, 1967, 23, 50-56.

\section{NOTES}

1. This research was supported in full by a U.S. Public Health Service, Department of Health, Education, and Welfare research grant from the National Institute of Neurological Diseases and Blindness. The authors wish to express their appreciation to Dr. Donald H. Eldredge for making available the facilities of the Electrophysiology Laboratory at Central Institue for the Deaf, for this work. We also thank Dr. Hallowell Davis and Dr. James D. Miller for their advice and criticism throughout the course of these experiments.

2. Present address: Bioacoustics Laboratory, School of Medicine, University of Pittsburgh, Pittsburgh, Pennsylvania 15213.

(Accepted for publication May 24, 1968.) 